To appear in "Magnetic Coupling between the Interior and the Atmosphere of the Sun", eds. S. S. Hasan and R. J. Rutten, Astrophysics and Space Science Proceedings, Springer-Verlag, Heidelberg, Berlin, 2009.

\title{
CME Observations from STEREO
}

\author{
N. Srivastava \\ Udaipur Solar Observatory, Physical Research Laboratory, Udaipur
}

\begin{abstract}
Summary. Coronal mass ejections (CMEs) are spectacular ejections of material from the Sun as seen in the coronal field of view. Regular observations are possible with both ground-based and space-based coronagraphs. I present our current understanding of CMEs based on multi-wavelength observations from groundbased instruments as well as from space missions such as SoHO. Based on the continuous and multi-wavelength observations of CMEs from SoHO over a period of more than a solar cycle, the physical properties of CMEs are described. Recent observations of CMEs with the SECCHI coronagraphs, namely COR1 and COR2 aboard the twin STEREO spacecrafts A and B are also presented. STEREO surpasses previous missions by providing a 3-D view of CME structure from two vantage points. Applications of STEREO observations to 3-D reconstructions of the leading edge of CMEs are described.
\end{abstract}

\section{Introduction}

Coronal mass ejections (CMEs) were first observed by OSO-7 in 1971 (Tousey 1973). Since then, a number of space-based and ground-based coronagraphs have been regularly recording images of the corona and CMEs. These observations have led to a fairly good general understanding of CMEs. They were traditionally observed in white light or continuum images by spacebased coronagraphs such as Solwind, the Solar Maximum Mission (SMM), and LASCO/C2\&C3, and with ground-based instruments such as the MK III and MK IV coronagraphs at Mauna Loa. However, CMEs have also been recorded in coronal emission lines for e.g., Fe XIV or Fe X, such as with the Norikura observatory and with LASCO-C1 onboard SoHO. Table 1 gives the field of view of various coronagraphs. SoHO was the first successful space mission with multiple instruments onboard to record various aspects of transient activity at multiple wavelengths. The Extreme Ultraviolet telescope (EIT) onboard SoHO not only can track down the source region of a CME, but SoHO also can record CME propagation through a field of view of $1-32 R_{\odot}$ with the 
Large Angle Spectrometric COronagraphs (LASCO) at an improved time cadence (Delaboudiniére et al. 1995; Brueckner et al. 1995). These continuous, multi-wavelength, and high time-cadence images have led to a better understanding of CME initiation and propagation. The details are presented in various reviews (Hudson et al. 2007; Schwenn et al. 2007).

An important research area in which SoHO observations proved to be extremely useful is space weather prediction. A large number of studies have been undertaken in this direction, in particular, for predicting the arrival time of CMEs at the Earth through continuous monitoring of the Sun and CME tracking. This helps in predicting the time of commencement of geomagnetic storms, which are normally expected to occur when a high-speed CME is directed towards the Earth and its magnetic field is oriented southward so as to ensure an effective coupling between the Earth's magnetic field and the propagating magnetic cloud. However, most prediction schemes used plane-of-sky speeds as proxies of radial speeds, which has led to large errors in the estimated arrival times (Schwenn et al. 2005). This error can be attributed to the lack of capability of SoHO to measure the radial speeds of CMEs. The problem is expected to be overcome with new observations from STEREO, which comprises twin spacecrafts with identical sets of instruments (Howard et al. 2008). The main goal of STEREO is to improve our understanding of the 3-D structure of CMEs with accurate and direct estimations of their true speeds and propagation directions.

Table 1. Comparison of different space-based coronagraphs used to record CMEs

\begin{tabular}{cccc}
\hline Coronagraph & Year & Field of view & Resolution \\
\hline OSO-7 & $1971-1973$ & $3.0-10.0 \mathrm{R}_{\odot}$ & $3^{\prime}$ \\
Skylab & $1973-1974$ & $2.0-6.0 \mathrm{R}_{\odot}$ & $5^{\prime \prime}$ \\
Solwind/P78-I & $1979-1985$ & $3.0-10.0 \mathrm{R}_{\odot}$ & Same as OSO \\
SMM & 1980 & $1.6-6.0 \mathrm{R}_{\odot}$ & $30^{\prime \prime}$ \\
& $1984-1989$ & & \\
LASCO & & & $11.2^{\prime \prime}$ \\
C1 & $1995-1998$ & $2.0-6.0 \mathrm{R}_{\odot}$ & $23.2^{\prime \prime}$ \\
C2 & $1995-$ & $3.7-32.0 \mathrm{R}_{\odot}$ & $112^{\prime \prime}$ \\
C3 & & & \\
SECCHI & $2006-$ & $1.4-4.0 \mathrm{R}_{\odot}$ & $7.5^{\prime \prime}$ \\
COR1 & & $2.0-15.0 \mathrm{R}_{\odot}$ & $14^{\prime \prime}$ \\
COR2 & & & \\
\hline
\end{tabular}

In what follows, statistical properties of CMEs are highlighted on the basis of SoHO observations and new observations from the SECCHI coronagraphs onboard the STEREO mission are discussed. In particular, I showcase the re- 
sults of 3-D reconstruction of a CME leading edge, using SECCHI-COR1 and COR2 observations, and discuss implications for space-weather prediction.

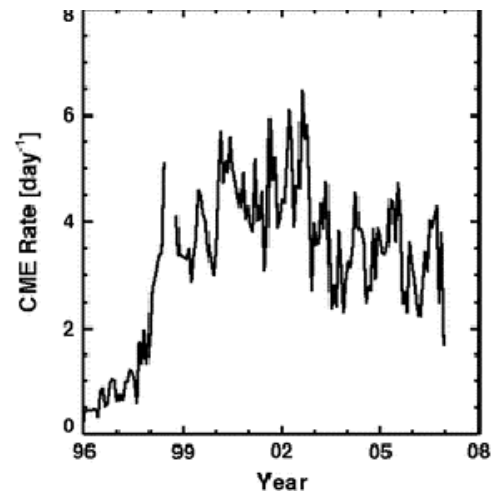

Fig. 1. The rate of occurrence of LASCO CMEs averaged over a full solar cycle, i.e., 1996-2007 (adapted from Gopalswamv et al. 2009).

\section{What have we learnt from LASCO observations?}

The three LASCO coronagraphs (Brueckner et al. 1995) onboard SoHO provide a nested field of view and have tracked a large number of CMEs from their launch at the solar surface. These observations confirm that CMEs generally have a three-part structure. They have a bright leading edge, a dark cavity believed to have a high magnetic field, followed by a bright and intense knot which mainly comprises prominence material. A large number of earthwarddirected CMEs were recorded by LASCO. These are observed as full halos in which brightness enhancement is seen in an angular span of $360^{\circ}$ around the occulter, while in the case of a partial halo, the brightness is seen over an angular span of more than $120^{\circ}$. The kinematics of full and partial halos have also been studied (Wang et al. 2002; Zhang et al. 2003; Zhao \& Webb 2003; dal Lago et al. 2004). These studies were aimed at inferring the travel time of the CMEs to the Earth. The estimated arrival time of a CME at the Earth is an important input for forecasting the time of occurrence of the resulting geomagnetic storms, provided there is a strong southward component of the propagating magnetic cloud.

A huge dataset has been collected with the LASCO coronagraphs over more than a solar cycle. It allows us to study CME properties over a large time period. The rate of occurrence of CMEs has been found to be 0.3 /day during the solar minimum around 1996, and it slowly increased to 5-6 CMEs/day during the solar maxima (Fig. (1). Yashiro et al. (2004) studied the variation of angular or apparent width of all CMEs that occurred during 1996-2003 and found that all non-halo CMEs have an average angular width of $47^{\circ}$ (left-hand panel of Fig. 21). The kinematics of the CMEs recorded by LASCO have also 

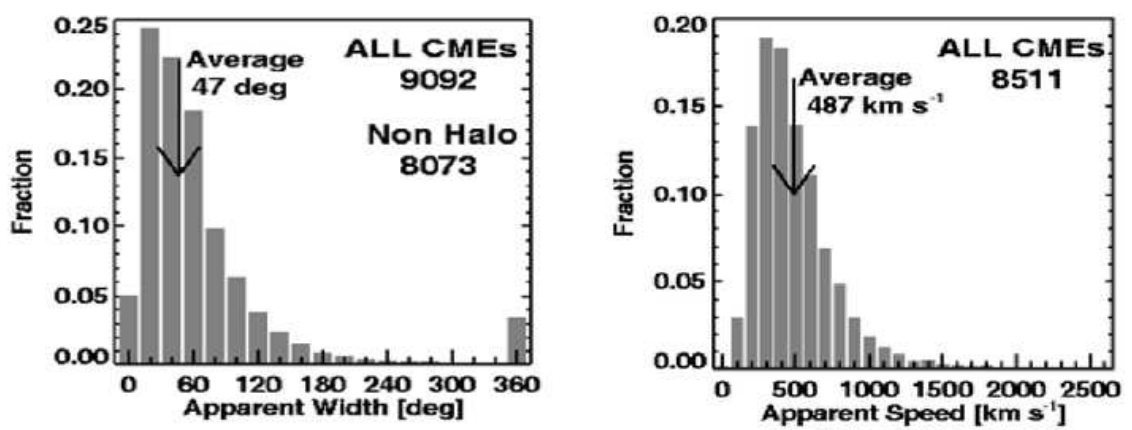

Fig. 2. Left: average width of all non-halo (angular width $0-120^{\circ}$ ) LASCO CMEs. Right: the projected speed of both halo and limb CMEs as recorded by the LASCO coronagraphs.

been studied by Yashiro et al. (2004). They measured the projected planeof-sky speeds of CMEs and found that these lie in the range $10-3000 \mathrm{~km} \mathrm{~s}^{-1}$, the average being $487 \mathrm{~km} \mathrm{~s}^{-1}$ (right-hand panel in Fig. 2). They also found that the average speed varies with the solar cycle, the average plane-of-sky speeds in the descending phase being lower than in the minimum.

The white-light coronal images have also been used to measure the density of CMEs by estimating their excess brightness. The density values thus obtained were used to derive the mass of CMEs, which ranges between $10^{15}$ $10^{16} \mathrm{~g}$. The average CME mass is smaller than the pre-SoHO values because LASCO could record low-mass CMEs owing to its high sensitivity. Usually, most CMEs show an initial increase in mass before reaching a constant value, as shown in Fig. 31 Yashiro et al. (2004) found that the mass estimates are uncertain by a factor of two. Using the estimated values of density and the measured values of the plane-of-sky speeds of the CMEs, the kinetic energies of CMEs were found to lie in the range $10^{31}-10^{32} \mathrm{erg}$. A comparison of statistical properties of white-light CMEs recorded by LASCO and recorded by earlier space-borne coronagraphs, namely, Solwind and SMM, is given in Table 2

A number of studies have been made on height-time profiles of various types of CMEs, indicating that there is broad spectrum of profiles. At one end of the speed spectrum, there are CMEs which rise gradually with slow speed $\left(50-100 \mathrm{~km} \mathrm{~s}^{-1}\right)$ over a period of several hours. They reach a terminal speed of $300-400 \mathrm{~km} \mathrm{~s}^{-1}$, equivalent to that of the slow solar wind speed at $20 \mathrm{R}_{\odot}$ (Srivastava et al. 1999; Srivastava et al. 2000). These are generally associated with eruptive filaments or prominences. At the other end of the speed spectrum are fast events, with speeds greater than $600 \mathrm{~km} \mathrm{~s}^{-1}$ which undergo maximum acceleration in the lower corona. Such CMEs are generally associated with flares (Zhang et al. 2004). 


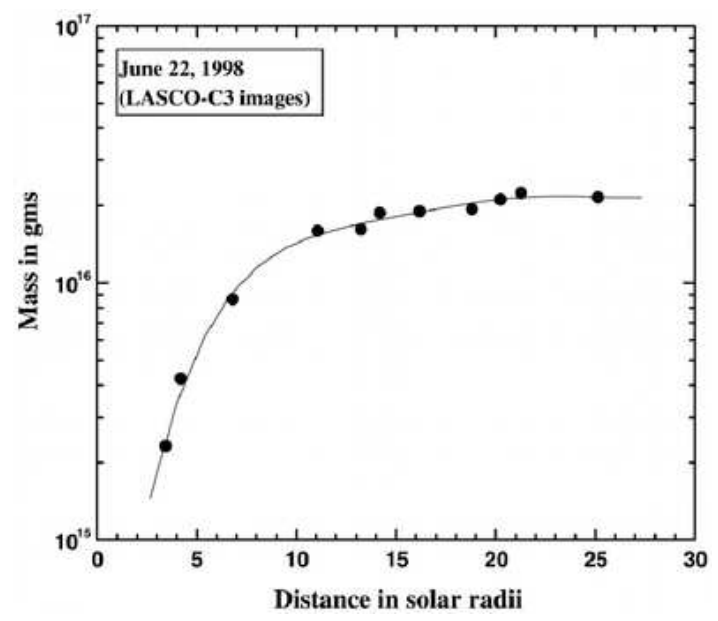

Fig. 3. The variation of total mass of the CME observed on June 21-22, 1998, with distance to the center of the Sun (adapted from Srivastava et al. 2000).

Table 2. Average CME properties

\begin{tabular}{|c|c|c|}
\hline Parameter & LASCO & Solwind/SMM \\
\hline observing duty cycle & $81.7 \%$ & $66.5 \%$ \\
\hline kinetic energy [erg] & $2.6 \times 10^{30}$ & $3.5 \times 10^{30}$ \\
\hline average mass $[\mathrm{gm}]$ & $1.4 \times 10^{15}$ & $4.1 \times 10^{15}$ \\
\hline mass flux [gm/day] & $2.7 \times 10^{15}$ & $7.5 \times 10^{15}$ \\
\hline average speed $\left[\mathrm{km} \mathrm{s}^{-1}\right]$ & 487 & 349 \\
\hline speed range $\left[\mathrm{km} \mathrm{s}^{-1}\right]$ & $10-3000$ & $80-1042$ \\
\hline \multicolumn{3}{|c|}{ rate of occurrence [CME/day] } \\
\hline cycle minimum & $0.31-0.77$ & 0.5 \\
\hline cycle maximum & $1.75-3.11$ & $5-6$ \\
\hline angular width $\left[^{\circ}\right]$ & 47 & 40 \\
\hline
\end{tabular}

\section{CME observations from SECCHI/STEREO coronagraphs}

The twin STEREO spacecrafts were launched during the solar minimum period in October 2006, when there was low expectation of the occurrence of CMEs. A preliminary study shows that the rate of CMEs observed with STEREO soon after its launch was $1 \mathrm{CME} /$ day, higher than the rate of LASCO CMEs recorded during solar minimum. COR1 data show that about 353 CMEs have been recorded until 30 January 2009, as shown at the COR1 website http://cor1.gsfc.nasa.gov/docs/prelim_events. The rate decreased to 0.5 CME/day in 2008, but shows a rising trend since then. Some of these CMEs could also be tracked in the outer corona with the COR2 and HI coronagraphs. As the STEREO observations provide simultaneous images of the corona from two vantage points, i.e., from "ahead" and "behind" space- 

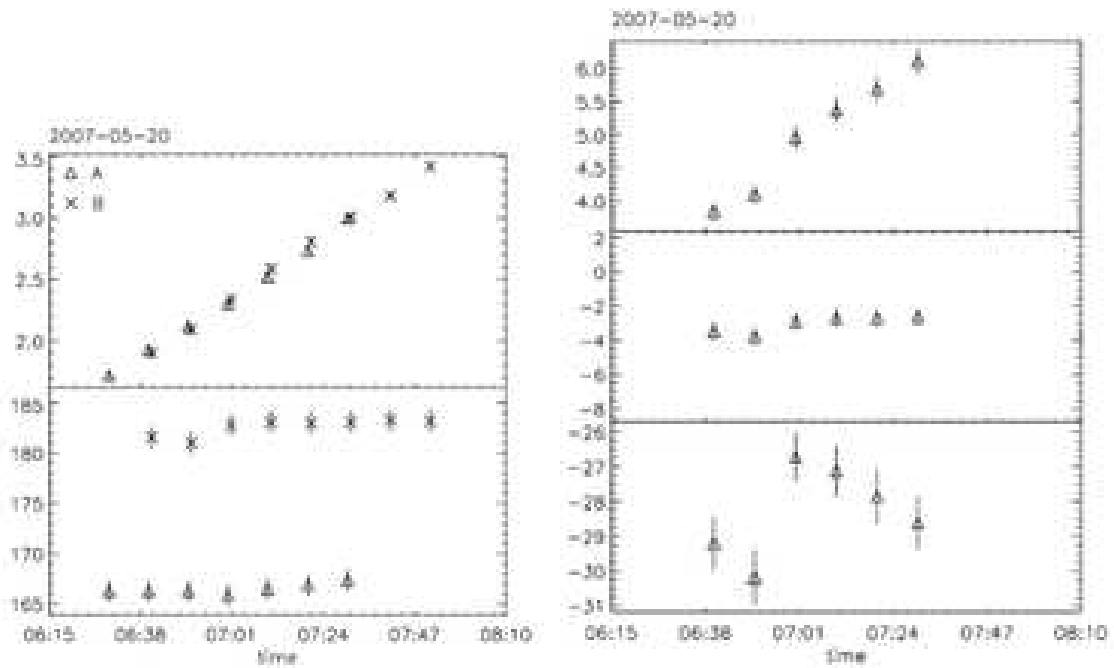

Fig. 4. Left: projected height (top) and position angle (bottom) of an identifiable feature along the leading edge of the 20 May 2007 CME, as observed in the COR1A and $\mathrm{B}$ coronagraphs. The position angle of the selected feature is measured counterclockwise from the North ecliptic pole. Right: true height (top), ecliptic longitude (middle), and ecliptic latitude (bottom) for the same feature, obtained using the height-time technique (adapted from Mierla et al. 2008).

crafts, they are useful to study the 3-D structure of CMEs. Prior to the launch of STEREO, different techniques were employed to derive the 3-D structure of solar features using SoHO data (Pizzo \& Biesecker 2004; Inhester 2006). The CME propagation properties were also derived by applying a cone model to the LASCO images (Zhao, Plunkett \& Liu 2002; Michalek et al. 2003; Michalek 2006). Other techniques that have been used for 3-D reconstruction are based on polarization measurements of the white light corona (Moran \& Davila 2004; Dere. Wang \& Howard 2005). Based on the findings of Schwenn et al. (2005) that the ratio between lateral expansion and radial propagation of CMEs is a constant, estimations of radial speeds, and hence the arrival time of CMEs at the Earth, were made.

Recently, with the launch of the twin spacecrafts STEREO A and B, disk observations of the solar atmosphere in extreme ultraviolet wavelengths (EUVI) and coronal observations in white light using the SECCHI coronagraphs from two vantage points simultaneously became available. This was used to study to study 3-D structure by reconstruction of solar features such as flare loops and CMEs. Using stereo pair images, one can also determine the true speeds and the directions of the leading edge and prominence of a CME. These are extremely valuable for space weather predictions, as one can not only estimate the true speeds and propagation direction of a CME in the corona, but also the exact arrival time at the Earth. A number of stud- 

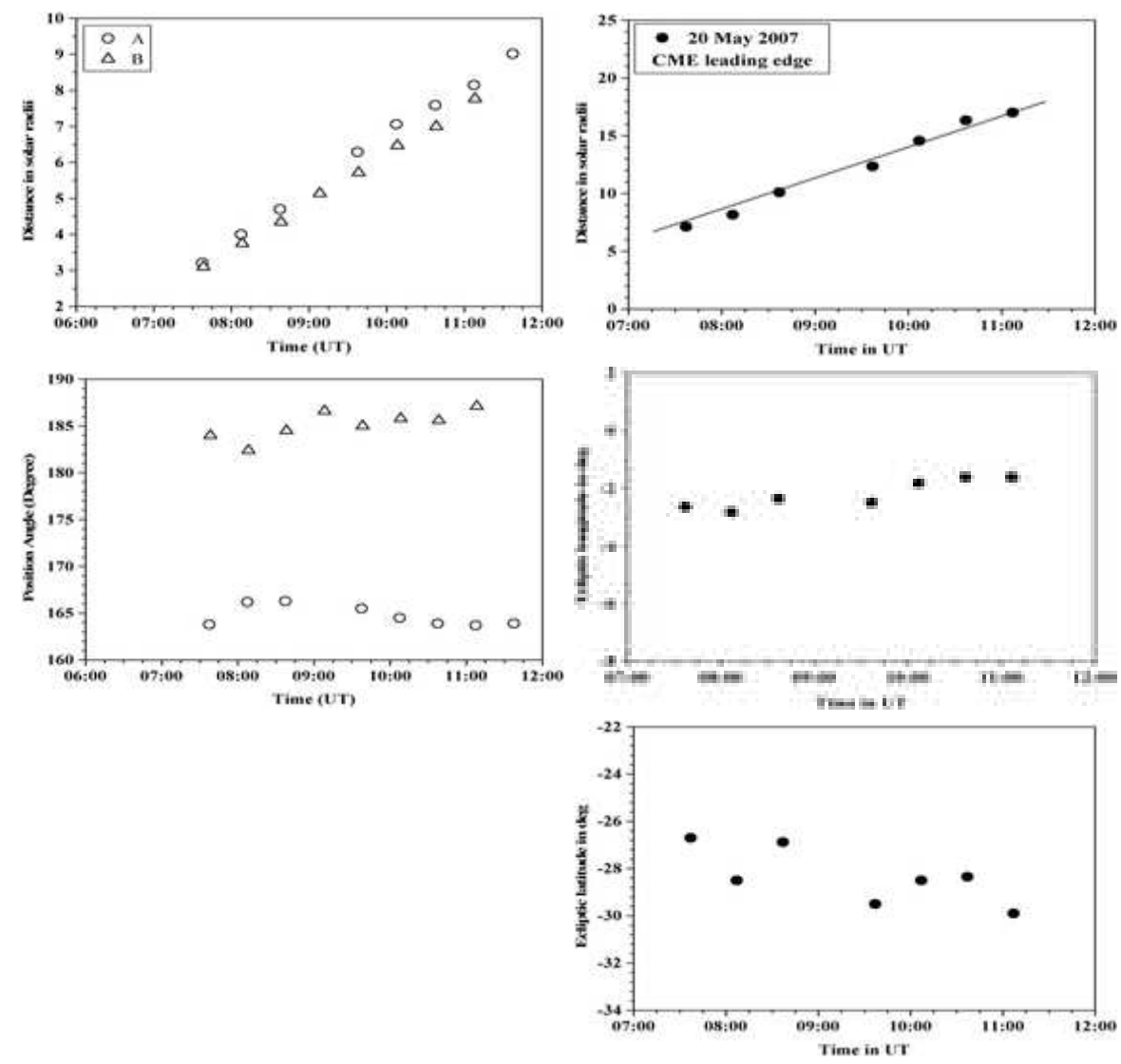

Fig. 5. Left: projected height (top) and position angle (bottom) of a selected feature along the leading edge of the 20 May $2007 \mathrm{CME}$, as observed in COR2A and B coronagraphs. Right: true height (top), ecliptic longitude (middle), and ecliptic latitude (bottom) for the same feature, obtained using the height-time technique for reconstruction.

ies in this direction using STEREO/SECCHI and EUVI images have been made recently. These studies are mainly based on tie-pointing reconstruction of STEREO images (Mierla et al. 2008; Mierla et al. 2009, Srivastava et al. 2009). The technique has proven to be extremely successful when applied to the leading edge of white light CMEs (Mierla et al. 2008; Mierla et al. 2009) and disk filament and loops (Gissot et al. 2008 and Aschwanden et al. 2008). Essentially, the tie-pointing technique for reconstructing CMEs is based on epipolar geometry, wherein the position of the two STEREO spacecrafts A and $\mathrm{B}$ and a point on the solar surface define a plane called the epipolar plane. The STEREO mission's plane is a special epipolar plane, passing through the Sun's centre and the two spacecrafts with its normal oriented towards the 
ecliptic North direction. The projections of all epipolar planes in the spacecraft's images are seen as epipolar lines in one stereoscopic image that passes through the same epipolar line in the other stereoscopic image. Tie-pointing involves finding a one-to one correspondence of a feature in both the stereo images along equal epipolar lines, calculating the line-of-sight ray that belongs to the respective images, and eventually constraining the rays to lie on the same epipolar plane (Trucco \& Verri 1998).

Table 3. Reconstruction of the leading edge of the 20 May $2007 \mathrm{CME}$

\begin{tabular}{llllllll}
\hline Method & Data & Projected & \multicolumn{3}{c}{ Reconstruction } & Remarks \\
& & & $\begin{array}{c}V_{\text {proj }} \\
{\left[\mathrm{km} \mathrm{s}^{-1}\right]}\end{array}$ & $\begin{array}{c}V_{\text {rec }} \\
{\left[\mathrm{km} \mathrm{s}^{-1}\right]}\end{array}$ & $\begin{array}{c}\lambda \\
{[\mathrm{deg}]}\end{array}$ & {$[\mathrm{deg}]$} & \\
\hline height-time & COR1 A\&B & 242 & 253 & 548 & -2 & -27 & Mierla et al. (2008) \\
& COR2 A\&B & 295 & 250 & 544 & -2 & -28 & this paper \\
tie-pointing & COR1 A\&B & & & 510 & 11 & -30 & Srivastava \\
& COR2 A\&B & & & & & et al. (2009) \\
\hline
\end{tabular}

A quick method based on tie-pointing is the height-time method for the 3-D reconstruction of CME features (Mierla et al. 2008). This is based on estimating the projected or "plane-of-sky" speeds of selected moving features of CMEs. Mierla et al. (2008) estimated the true heights, speeds and directions of the leading edges of three CMEs using COR1 coronagraphic images. An example of 3-D reconstruction of one of the CMEs dated 20 May 2007 studied in their paper is shown in Fig. 4. By applying the height-time method to COR1 images, Mierla et al. (2008) found that the 20 May 2007 CME was located at ecliptic longitude and latitude of around $-2^{\circ}$ and $-27^{\circ}$ (i.e., south of the ecliptic), respectively. The plane-of-sky speeds as measured from the COR1 A and COR1 B coronagraphs were estimated to be approximately 242 and $253 \mathrm{~km} \mathrm{~s}^{-1}$. The true speed was estimated to be approximately $548 \mathrm{~km} \mathrm{~s}^{-1}$. In this contribution, we extended the analysis to COR2 images which cover a field of view from $2-15 \mathrm{R}_{\odot}$. Using the stereo pair images of the COR2 coronagraphs and applying height-time analysis on the same feature as in the COR1 images, we obtained the projected speed as approximately 298 and $250 \mathrm{~km} \mathrm{~s}^{-1}$ in the A and B images, respectively (Fig. 5). The reconstructed speed was estimated at $544 \mathrm{~km} \mathrm{~s}^{-1}$. The average ecliptic longitude and latitude were estimated to be $-2^{\circ}$ and $-28^{\circ}$ respectively. A comparison of the reconstruction parameters obtained using different techniques for the leading edge of the May 20, $2007 \mathrm{CME}$ is given in Table 3. The projected speeds $\left(V_{\text {proj }}\right)$ of the leading edge are given in the third column; the reconstructed parameters i.e., the reconstructed speed $\left(V_{\text {rec }}\right)$, the ecliptic longitude $(\lambda)$, and the ecliptic latitude $(\theta)$ of the identified feature along the leading edge are given in the fourth column. The table shows that the results from 
the different techniques are in good agreement. This implies that the leading edge moves at almost constant speed, with only small deflections in the direction of propagation.

A magnetic cloud was found to be associated with the CME of 20 May 2007 (Kilpua et al. 2008). This cloud arrived at the STEREO A spacecraft at 00:26 UT on May 23. Thus, the actual travel time of the CME to the Earth was 68 hours. In addition, the measured speed of the magnetic cloud at STEREO A was $535 \mathrm{~km} \mathrm{~s}^{-1}$. Assuming that this speed is the average speed at which the CME travelled towards the Earth, the predicted travel time is about 74 hours, which is equal to the actual arrival time within the measurement errors. It should be emphasized that use of the projected speeds would yield large errors in the estimation of the travel time. Thus, 3-D reconstruction using STEREO pair images has an important bearing on space weather prediction (Srivastava et al. 2009).

\section{Summary and conclusion}

SECCHI observations have surpassed the capabilities of SoHO observations. With new views of CMEs obtained by applying reconstruction techniques to the stereo image pairs, our knowledge of the 3-D structure and propagation of CMEs will advance considerably. Also, with reliable estimations of true CME speeds and propagation directions, the solar community is poised to achieve unprecedented success in space weather prediction.

Acknowledgement. The author acknowledges the help of STEREO/SECCHI consortia for providing the data used and presented in this paper. The author also thanks M. Mierla (Royal Observatory of Belgium) and B. Inhester of MPS, Germany, for fruitful discussions on CME reconstruction using COR1 and COR2 images.

\section{References}

Aschwanden, M., Wlser, J.-P., Nitta, N. V., Lemen, J. R. 2008, ApJ, 679, 827

Brueckner, G. E., Howard, R. A., Koomen, M. J., Korendyke, C. M., Michels, D. J., Moses, J. D., et al. 1995, Solar Phys., 162, 357

dal Lago, A. Vieira, L. E. A., Echer, E., Gonzalez W. D., 2004, Solar Phys., 222, 323

Delaboudiniére, J.-P., Artzner, G. E., Brunaud, J., Gabriel, A. H., Hochedez, J. F., Millier, F., Song, X. Y., Au, B., Dere, K. P., Howard, R. A., and 18 coauthors, Solar Phys., 162, 291

Dere, K. P., Wang, X., Howard, R. 2005, ApJ, 620, L119.

Gissot, S. F., Hochedez, J.-F., Chainais, P., Antoine, J.-P., Solar Phys., 252,397

Gopalswamy, N., Yashiro, S., Michalek, G., Stenborg, G., Vourlidas, A., Freeland, S., Howard, R. 2009, Earth, Moon and Planets, doi 10.1007/s11038-008-9282-7

Hapgood, M. A. 1992, Plan. Space Science, 40, 711 
Howard, R.A., Moses, J.D., Vourlidas, A., Newmark, J.S., Socker, D.G., Plunkett, S.P., et al. 2008, Space Sci. Rev., 136, 67

Hudson et al. in Coronal Mass Ejections eds. H. Kunow, N.U. Crooker, J.A. Linker, R. Schwenn, \& R. Von Steiger, Space Science Series of ISSI 2007, 13

Inhester, B. 2006, Publ. Int. Space Sci. Inst., astro-ph/0612649 in press

Kaiser, M. L., Kucera, T. A., Davila, J. M., St. Cyr, O. C., Guhathakurta, M., Christian, E. 2008, Space Sci. Rev., 136, 5.

Kilpua, E. K. J., Liewer, P. C., Farrugia, C., Luhmann, J. G., Moest, C., Li, Y., Liu, Y., Lynch, B. J., Russell, C. T., Vourlidas, A., Acuna, M. H., Galvin, A. B., Larosn, D., Sayvaud, J. A. 2008, Solar Phys., in press

Michalek, G., Gopalswamy, N., Yashiro, S. 2003, ApJ, 584, 472

Michalek, G. 2006, Solar Phys., 237, 101

Mierla, M. Davila, J., Thompson, W., Inhester, B., Srivastava, N., Kramar, M., St. Cyr. O. C., Stenborg, G, Howard, R. A. 2008,Solar Phys., 252, 385

Mierla, M., Inhester, B., Marque, C., Rodriguez, L., Gissot, S., Zhukov, A., Berghmans, D., Davila, J. 2009, Solar Phys., submitted

Moran, T.G., Davila, J. 2004, Science, 305, 66

Pizzo, V.J., Biesecker, D.A. 2004, Geophys. Res. Lett., 31, L21802

Schwenn, R, Dal Lago, A., Huttunen, E., Gonzalez, W.D., 2005, Ann. Geophys., 23, 1033

Schwenn et al. in Coronal Mass Ejections eds. H. Kunow, N.U. Crooker, J.A. Linker, R. Schwenn, \& R. Von Steiger, 2007, Springer, Berlin, 137

Srivastava, N., Schwenn, R., Inhester, B., Stenborg, G., \& Podlipnik, B. 1999, in Solar Wind 9, AIP Conf. Proc. 471, 115

Srivastava, N., Schwenn, R., Inhester, B., Martin, S. F., Hanaoka, Y. 2000, ApJ, 534,468

Srivastava, N., Inhester, B., Mierla, M., Podlipnik, B. 2009, Solar Phys., submitted

St. Cyr, O. C., Howard, R. A., Sheeley, N. R., Plunkett, S. P., Michels, D. J., Paswaters, S. E., Koomen, M. J., Simnett, G. M., Thompson, B. J., Gurman, J. B., Schwenn, R., Webb, D. F., Hildner, E., Lamy, P. L. 2000, J. Geophys. Res., 105, 18169

Thompson, W. T., Davila, J. M., Fisher, R. R., Orwig, L. E., Mentzell, J. E., Hetherington, S.E. 2003, in Keil, S. L., Avakyan, S. V. (eds.), Innovative Telescopes and Instrumentation for Solar Astrophysics, SPIE, 4853, 1

Thompson, W.T. 2006, A\&A, 449, 791

Tousey R., The Solar Corona, in Space Research XIII 1973, eds. M. J. Rycroft, S. K. Runcorn, 713, Akademie-Verlag, Berlin

Trucco, E., Verri, A. 1998, Introductory Techniques for 3-D Computer Vision, Prentice Hall

Vourlidas, A., Buzasi, D., Howard, R. A., Esfandiari, E. in: Solar variability: from core to outer frontiers. The 10th European Solar Physics Meeting, ed. A. Wilson, ESA SP-506, 91

Wang, Y. M., Ye, P. Z., Wang, S., Zhou, G. P., Wang, J. X. 2002, J. Geophys. Res., 107,2

Xie, H., Ofman, L., Lawrence, G. 2004, J. Geophys. Res., 109, A03109.

Yashiro, S., Gopalswamy, N., Michalek, G., St. Cyr, O. C., Plunkett, S. P., Rich, N. B., Howard, R. A. 2004, J. Geophys. Res., 109, A07105.

Yurchyshyn, V., Wang, H., Abramenko, V. 2003, Adv. Sp. Res., 32, 1965

Zhang, J., Dere, K. P., Howard, R. A., Bothmer, V. 2003, ApJ, 582, 520 
Zhang, J., Dere, K. P., Howard, R. A., Vourlidas, A., 2004, ApJ, 604, 420

Zhao, X. P., Plunkett, S. P., Liu, W. 2002, J. Geophys. Res., 107, 1223

Zhao, X. P., Webb, D. F. 2003, J. Geophys. Res., 108, 4 